\title{
Concurrent On-the-fly SCC Detection for Automata-based Model Checking with Fairness Assumption
}

\author{
Zhimin $\mathrm{Wu}^{*}$, Yi Xu*, Akin Gunay* Yang Liu*, Shengchao Qin ${ }^{\ddagger}$ \\ *Nanyang Technological University, Singapore \\ ${ }_{\ddagger}^{\ddagger}$ Teesside University, United Kindom
}

\begin{abstract}
Model checking is an automated technique for verifying temporal logic properties of finite state systems. Tarjan's algorithm for detecting Strongly Connected Components (SCCs) is a widely used depth-first search procedure for Automatabased (LTL) model checking. It works on the SCC detection on-the-fly with the composition of transition systems and Büchi Automaton (state space generation), which has been deployed as sequential implementations in many tools. However, these implementations suffer from heavy time cost for systems which involve a large number of SCC explorations. To address this issue, in this paper, we develop a concurrent SCC detection approach for the on-the-fly generated state space in LTL model checking by expanding the existing concurrent Tarjan's algorithm. Besides, we involve fairness checking. Different that the previous work, which performs fairness checking after the generation of a complete SCC, in our approach we perform fairness checking during SCC generation to improve efficiency. We implement our approach in PAT model checker. Our experimental results show that our approach achieves up to $2 \mathrm{X}$ speedup for the complete SCC detection in large-scale system models compared to the sequential on-the-fly model checking in PAT. Besides, our parallel on-the-fly fairness checking approach speedups fairness checking around $2 X \sim 45 X$.
\end{abstract}

\section{INTRODUCTION}

Automata-based LTL model checking is emptiness checking of the composition of a transition system $\mathcal{M}$ and a Büchi Automaton $\mathcal{B}_{\neg \varphi}$, which represents the negation of an LTL property $\varphi$ [2]. The composition process indicates that the state space is unknown in advance, which is on-the-fly generated. The idea of emptiness checking is to search the on-the-fly generated state space to find an execution path that is accepted by the Büchi automaton. Strongly Connected Component (SCC) based model checking that uses Tarjan's algorithm [18] for SCC detection, is a well-known approach for LTL model checking. In this approach, the problem of LTL model checking is converted to the detection of an infinite path that is accepted by the Büchi Automaton. The infinite path contains SCCs with accepting cycles. It uses depth-frist search to explore the state space to detect SCC.

Fairness and liveness are two essential notions for faithfully modeling the execution progress of a process in a collection of concurrent processes [14]. Fairness constraints can be expressed in LTL. Thus, fairness checking can be integrated into SCC-based LTL model checking, which expand the original process to the verification of the fairness assumption's satisfaction in all detected SCCs.
There are many sequential implementations of SCC detection for automata-based LTL model checking with fairness assumption. However, besides the state space explosion problem [4], sequential implementations of automata-based LTL model checking with fairness do not scale well for large scale systems with a large number of SCCs. In Automatabased LTL model checking with fairness, the verification process is overlapped with the state space generation, which is the product of $\mathcal{M}$ and $\mathcal{B}_{\neg \varphi}$. The verification process may immediately reports failure, if it detects a fair SCC. While sometimes the verification process has heavy costs, since the fair SCC does not exist or appear after a large number of SCCs being detected. In this paper, we extend the concurrent implementation [11] of Tarjan's algorithm based on known state space to concurrent SCC detection for LTL model checking with on-the-fly generated state space. We aim to improve the verification performance. Furthermore, we develop an efficient approach for parallel fairness checking.

The main challenges of utilizing parallel computing to accelerate Automata-based LTL model checking with fairness are: (1) Tarjan's algorithm is a DFS process, which has attracted many researches on its parallelization. However, all researches work on the complete state space. It is challenging and significant to convert a parallel Tarjan's algorithm to be available for on-the-fly generated state space. 2) An efficient data distribution approach is necessary since the state space is unknown in advance. It is also important to maintain data consistency. 3) The sequential algorithm mentioned in [21] verifies the fairness after the generation of a complete SCC, which can be regarded as an independent part from the verification process. A better way for fairness checking is promising.

To solve these challenges, we expand the concurrent Tarjan's algorithm from Lowe [11] to fulfil the Automatabased LTL model checking for SCC detection on on-the-fly generated state space. Our key contributions in this paper are as follows: 1) Lowe's Tarjan's algorithm depends on complete state space (unrooted mode). Although he mentioned rooted mode in both [12] and [11], there is no details or experiments for this. We expand the algorithm to fit the on-the-fly state space generation. Based on it, we build the parallel approach to cover the features of on-the-fly SCC-based LTL model checking. 2) We build our own data distribution rules for onthe-fly generated state space. 3) We design and develop an efficient on-the-fly parallel fairness checking approach, which performs the fairness checking during the generation of SCC 
instead of performing it separately after the generation like [21] does. 4) We implement our approach in the Process Analysis Toolkit (PAT) [17] and make it work on a wide range of system models. Our evaluation shows that after integrating our approach, we achieve a $2 \mathrm{X}$ performance improvement in LTL model checking which involve the exploration of a large number of SCCs. And 2X 45X speedup for fairness checking.

The structure of this paper is as follows: In Section II, we introduce the background and related work, including the concurrent Tarjan's algorithm from Lowe [11]. In Section III, we present our design of the concurrent on-the-fly SCC detection for automata based (LTL) model checking with fairness assumption. In Section IV we present our experiments and evaluation. Finally, we present the conclusion and future work in Section V.

\section{BACKGROUND AND RELATED WORK}

\section{A. Automata-based Model Checking for LTL}

Given a system model $M$ and an LTL property $\varphi$, model checking is to check $M=\varphi$. The model of concurrent systems $M_{c}$ can be represented as a composition of several interleaving processes, each of which is expressed as labelled transition systems.

Definition 1: Given a set of invisible and visible events $\Sigma$, a labelled transition system (LTS) is a 3-tuple $M=\left(S, s_{0}, \rightarrow\right)$ where $S$ is a set of states, $s_{0} \in S$ is the initial state, $\rightarrow \subseteq$ $S \times \Sigma \times S$ is a transition relation.

In Automata-based model checking, the negation of $\varphi$ is expressed in an equivalent Büchi automaton $\mathcal{B}_{\neg \varphi}$ (Def. 2), which is then composed with the LTS representing the system model. The composition process, formal defined in Def. 3, generates the state space of $M_{c}$. Each state in the state space of $M_{c}$ is a meta-state (i.e., a vector of states), which we define as $s v=S \times S_{\mathcal{B}}$, where $n \in 1 . . n, S_{\mathcal{B}} \in \mathcal{B}_{\neg \varphi}$. Then, checking $M_{c}=\varphi$ can be done as checking of the emptiness of the product between $M_{c}$ and $\mathcal{B}_{\neg \varphi}$. SCC detection is a key subroutine in this process.

Definition 2: A Büchi Automaton is a tuple $\mathcal{A}=$ $\left(\Sigma_{\mathcal{B}}, S_{\mathcal{B}}, \rho, b_{0}, F\right)$, where $\Sigma$ is an alphabet, $S_{\mathcal{B}}$ is a set of Büchi states, $\rho: S_{\mathcal{B}} \times \Sigma$ is a nondeterministic transition function, $b_{0} \in S_{\mathcal{B}}$ is an initial state, and $F \subseteq B$ is a set of accepting states.

Definition 3: Given a LTS representing the system model $M=\left(S, s_{0}, \rightarrow\right)$ and a set of alphabets (events) $\Sigma$, a Büchi Automaton $\mathcal{B}_{\neg \varphi}$, the composition of $M$ and $\mathcal{B}_{\neg \varphi}$ is a transition system: $\left.M \times \mathcal{B}_{\neg \varphi}=\left(S \times B,\left(s_{0}, b_{0}\right)\right), \rightarrow\right)$. The corresponding set of events is $\Sigma \cup \Sigma_{\mathcal{B}}$. The transition relation after the parallel composition is the smallest transition relation which satisfies the following:

$$
\begin{array}{ll} 
& \left(\left(s_{1}, b_{1}\right), \alpha,\left(s_{1}^{\prime}, b_{1}^{\prime}\right)\right) \in \rightarrow \text { if }\left(s_{1}, \alpha, s_{1}^{\prime}\right) \in \rightarrow \wedge \ldots \wedge \\
& \left(s_{n}, \alpha, b_{n}^{\prime} \in \rightarrow\right) . \\
\text { 2) } & \left(\left(s_{1}, b_{1}\right), \alpha,\left(s_{1}^{\prime}, b_{1}\right)\right) \in \rightarrow \text { if }\left(s_{1}, \alpha, s_{1}^{\prime}\right) \in \rightarrow \wedge \alpha \notin \Sigma_{\mathcal{B}} \\
3) \quad\left(\left(s_{1}, b_{1}\right), \alpha,\left(s_{1}, b_{1}^{\prime}\right)\right) \in \rightarrow \text { if }\left(b_{1}, \alpha, b_{1}^{\prime}\right) \in \rightarrow \wedge \alpha \notin \Sigma .
\end{array}
$$

Scalability of LTL model checking suffers from state space explosion [4]. On-the-fly model checking [5] checks the emptiness while constructing the product state space of the

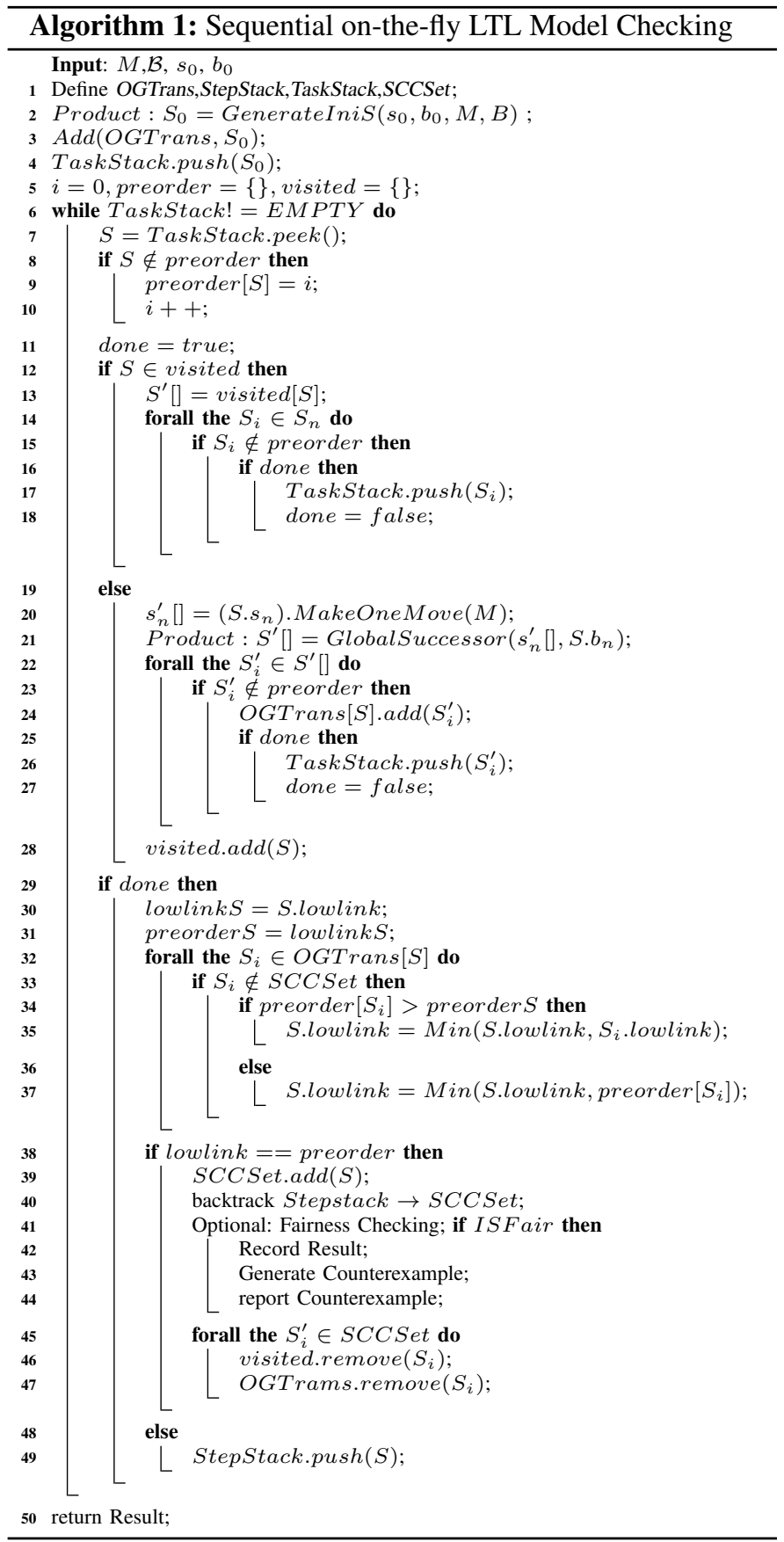

interleaving processes. In this context, Automata-based LTL model checking does SCC exploration on the composition of $\varphi$ and $\mathcal{B}_{\neg \varphi}$ with Tarjan's algorithm. The sequential implementation of this process is shown in Algorithm, which is the version in PAT [21].

\section{B. Fairness Assumption and Model Checking with Fairness}

Fairness is typically needed to prove liveness, which is concerned with a fair resolution of nondeterminism [13]. Without fairness, liveness verification may output unrealistic loops during which one process or event is infinitely ignored by the scheduler or one processor is infinitely faster than others [16]. 
Given a LTS $M=\left(S, s_{0}, \rightarrow\right)$ with event set $\Sigma$ we first provide the following definitions on events and processes, where $\alpha$ is a typical element of $\Sigma$ : (1) enabledEvt $(s)$ is the set of enabled events at $s \in S$ such that $\left\{\alpha \mid\left(s, \alpha, s^{\prime}\right) \in \rightarrow\right\}$. (2) enabledProc $(s)$ is the set of enabled processes at $s$. That is, if a process $p \in$ enablePro $(s)$, it can progress in the system state $s$. (3) engagedEvt $\left(s, \alpha, s^{\prime}\right)$ indicates the engaged event $\alpha$ (4) engagedProc $\left(s, \alpha, s^{\prime}\right)$ indicates the set of processes which progress via the transition $\left(s, \alpha, s^{\prime}\right) \in \rightarrow$.

Given an execution $R_{i}^{j}$,

$R_{i}^{j}=\left(\left(s_{0}, b_{0}\right), \alpha_{0}, \ldots\left(s_{i}, b_{i}\right), \alpha_{i} \ldots\left(s_{j}, b_{j}\right), \alpha_{j},\left(s_{j+1}, b_{j+1}\right)\right)$

where $s_{j} \in S$ and $b_{j} \in \mathcal{A}_{\neg \varphi}$. There is an SCC in this execution which indicates $s_{i}=s_{j+1}, b_{i}=b_{j+1}$. If $\left(b_{0}, b_{1} \ldots b_{k} \ldots\right)$ is accepting to $\mathcal{A}_{\neg \varphi}, R_{i}^{j}$ is accepting. With specific fairness assumption, $R_{i}^{j}$ is fair if and only if $\left(s_{0}, \alpha_{0}, s_{1}, \alpha_{1} \ldots s_{k}, \alpha_{k} \ldots\right)$ is fair. Several more definitions are shown: (1) alwaysEvt $\left(\left(R_{i}\right)^{j}\right)=\{\alpha \mid \forall k$ : $\{i . . j\}, \alpha \in$ enabledEvt $\left.\left(s_{k}\right)\right\}$ (2) alwaysPro $\left(\left(R_{i}\right)^{j}\right)=\{p \mid \forall k$ : $\{i . . j\}, p \in$ enabledPro $\left.\left(s_{k}\right)\right\}$. (3) onceEvt $\left(\left(R_{i}\right)^{j}\right)=\{\alpha \mid$ $\exists k:\{i . . j\}, \alpha \in$ enabledEvt $\left.\left(s_{k}\right)\right\}$. (4)oncePro $\left(\left(R_{i}\right)^{j}\right)=\{p \mid \exists k$ : $\{i . . j\}, p \in$ enabledPro $\left.\left(s_{k}\right)\right\}$.

We consider five categories of fairness assumptions: (1) Strong Global Fairness (SGF): An execution satisfies SGF if and only if for all $\left(s, \alpha, s^{\prime}\right) \in \rightarrow$, if $s=s_{i}$ for infinitely many $i, s_{i}=s$ and $\alpha_{i}=\alpha$ and $s_{i+1}=s^{\prime}$ for infinitely many $i$. (2) Event-level Strong Fairness (ESF): An execution $R$ satisfies ESF if and only if for all events $\alpha$, if $\alpha$ is infinitely often enabled, then $\alpha_{i}=\alpha$ for infinitely many $i$. (3) Event-level Weak Fairness (EWF) [9]: An execution $R$ satisfies EWF if and only if for all $\alpha$, if $\alpha$ finally becomes enabled forever in $R$, then $\alpha_{i}=\alpha$ for infinitely many $i$. (4) Process-level Strong Fairness (PSF): An execution satisfies PSF if and only if for all processes $p$, if $p$ is infinitely often enabled, then $p \in$ engagedProc $\left(s_{i}, \alpha_{i}, s_{i+1}\right)$ for infinitely many i. (5) Processlevel Weak Fairness $(P W F)$ : An execution $E$ satisfies PWF if and only if for every processes $p$, if $p$ eventually becomes enabled forever in $E$, then $p \in \operatorname{engagedProc}\left(s_{i}, \alpha_{i}, s_{i+1}\right)$ for infinitely many $i$.

In particular, for automaton-based model checking with fairness assumption, we always build the LTL formula with fairness constraints. But the size of the Büchi automaton is exponential to the size of the LTL formula, which makes it infeasible to handle large formulas. e.g., formulas with many fairness constraints. Thus, in this paper, our sequential SCCbased LTL model checking with fairness algorithm adopts the solution of PAT [17] and handles the fairness checking on the generated SCC as follows. Given an SCC $S$ in the product of $M$ and $\mathcal{A}_{\neg \varphi}$ and fairness assumption $\mathcal{F}$, if there is no such $S$ that $S$ is accepting and:

1) $\quad$ onceEvt $(S) \subseteq$ engagedEvt $(S) \Longleftrightarrow M$ satisfies $F_{E S F}$.

2) alwaysEvt $(S) \subseteq$ engagedEvt $(S) \Longleftrightarrow M$ satisfies $F_{E S F}$.

3) $\quad$ oncePro $(S) \subseteq$ engagedPro $(S) \Longleftrightarrow M$ satisfies $F_{P S F}$.

4) alwaysPro $(\bar{S}) \subseteq$ engagedPro $(S) \Longleftrightarrow M$ satisfies $F_{P W F}$.

\section{Related work}

Parallel computing has been widely used to deal with model checking problems. [1] presents the GPU acceler- ated state space generation. Our previous work [19] presents the GPU-based counterexample generation for LTL model checking. [20] presents the GPU-based on-the-fly reachability checking. [7] and [8] present a multicore NDFS algorithm for LTL model checking. [10] proposes a parallel LTL model checking algorithm which starts multiple threads to generate the SCC when it is detected, and the fairness checking occurs in the thread for SCC generation. [6] presents a state compression and reconstruction approach. It builds a state space exploration algorithm based on the shared memory multicore architecture. [3] presents a novel emptiness checking approach for LTL model checking, which is based on SCC enumeration and support TGBA. Its key feature it the usage of a global union-find data structure. [15] presents a parallel SCC decomposition based on the set-based SCC algorithms instead of Tarjan's algorithm. [11] introduces some concurrent DFS-based algorithms, such as concurrent Tarjan's algorithm for SCC detection. It is the main related work for our approach. We describe it in detail below.

Concurrent Tarjan's Algorithm: Lowe [11] design a concurrent Tarjan's algorithm for SCC detection. Given a system model $M$ with complete state space, the SCC exploration starts from multiple different states. They define an object Search as the unit for exploration, and an object Scheduler for the arrangement of searches to threads. Compared to traditional Tarjan's algorithm, Lowe's approach differs in three parts: 1) If a state is visited, it should record the ID of the corresponding Search. A state can only be visited by one Search. 2) If a Search $i$ explores a state that records other Search's ID $j$, $i$ is suspend to $j$. 3) The suspended relation can be broken when the status of the state becomes completed, which means the state has been detected to be in one SCC. One iteration of the overall process is shown in Fig. 1: Each Search has its own Taskstack and Stepstack. It follows the sequential Tarjan's algorithm to explore the successors (child) of its initial state. Then based on the description above, they introduce a SuspendingRealtion set. Searches need to check if there is a cycle in the SuspendingRelation, shown in part $A$ in Fig. 2. If so, the states in the Searches that related to this cycle are transferred to one single Search to block this cycle, shown in part B, Fig. 2. This cycle is also an SCC. More details can be found in [11]. When any search finds an SCC, all states in SCC should be marked as completed to activate the Searches which are suspended.

The difference between our work and Lowe's is that we expand the concurrent SCC detection for automata-based LTL model checking with fairness assumption based on the concurrent Tarjan's algorithm. We support the concurrent onthe-fly SCC detection, in which the state space is unknown in advance. Although Lowe mentioned this mode (rooted mode) in both [11] and [12], he doesn't supply details or experiments. We also support the parallel on-the-fly fairness checking.

\section{CONCURRENT ON-THE-FLY SCC DETECTION FOR MODEL CHECKING UNDER FAIRNESS ASSUMPTION}

The core in the emptiness checking of automata-based model checking is SCC detection. Thus, our approach is based on the concurrent Tarjan's algorithm (Section II-C). Our target is to expand it to construct a concurrent version for the onthe-fly generated state space. To this end, we first present 


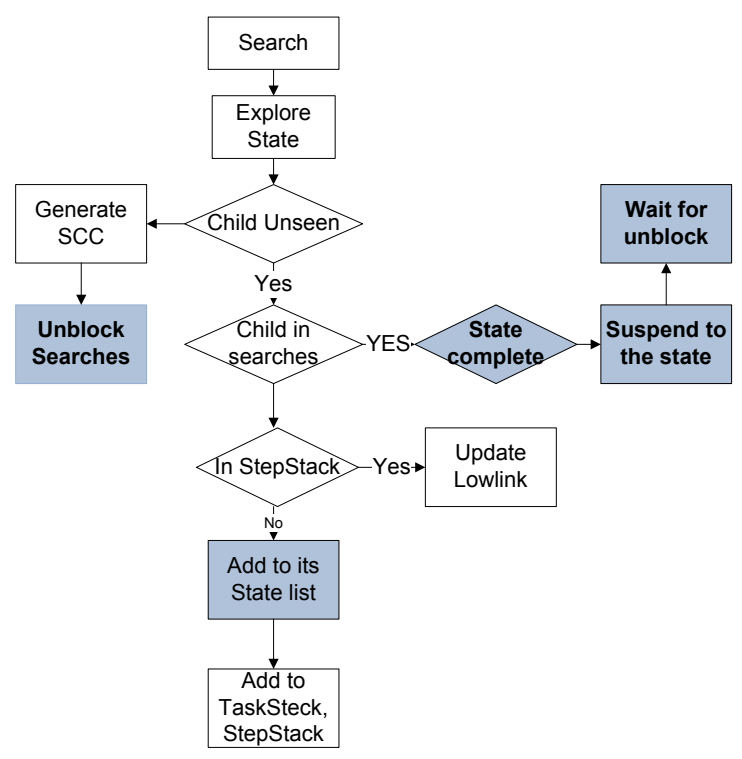

Figure 1. Execution Process in One Iteration

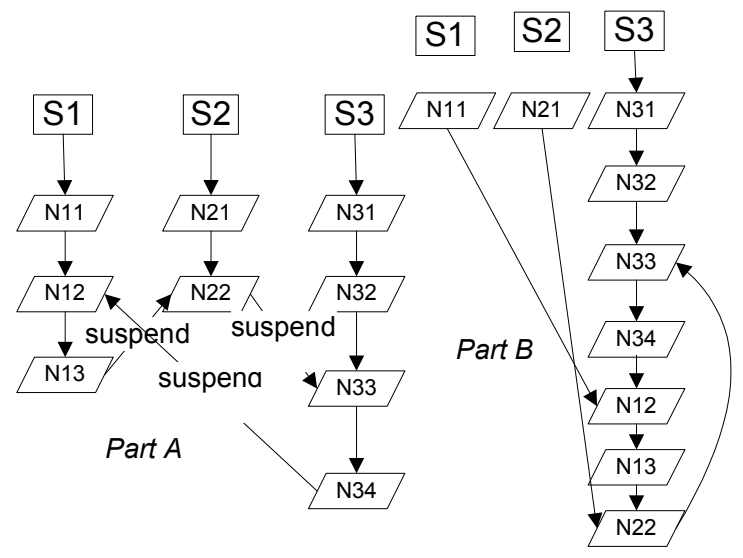

Figure 2. Blocking Cycle

several key challenges, which indicate the efforts on making the concurrent Tarjan's algorithm feasible for automata-based model checking with on-the-fly generated state space: (1) In this context, the state space is generated by the composition of transition system and büchi automaton. Thus, the state space is unknown in advance, and the simple predefined ID for each state in the concurrent Tarjan's algorithm is not available. (2) In concurrent Tarjan's algorithm, if a state is explored in a search, there is no duplicate state being explored in any other searches since the state space is known in advance. However, duplicate elimination is an important problem for concurrent on-the-fly generated state space. If there are duplicate states, the judgment on the suspension of a search cannot be made correctly. (3) Preservation of data consistency in the synchronization process is another challenge for concurrent SCC detection on on-the-fly generated state space, which require upgrading of the execution process and data structures.

In this section, we present how our approach solves the above challenges in detail.

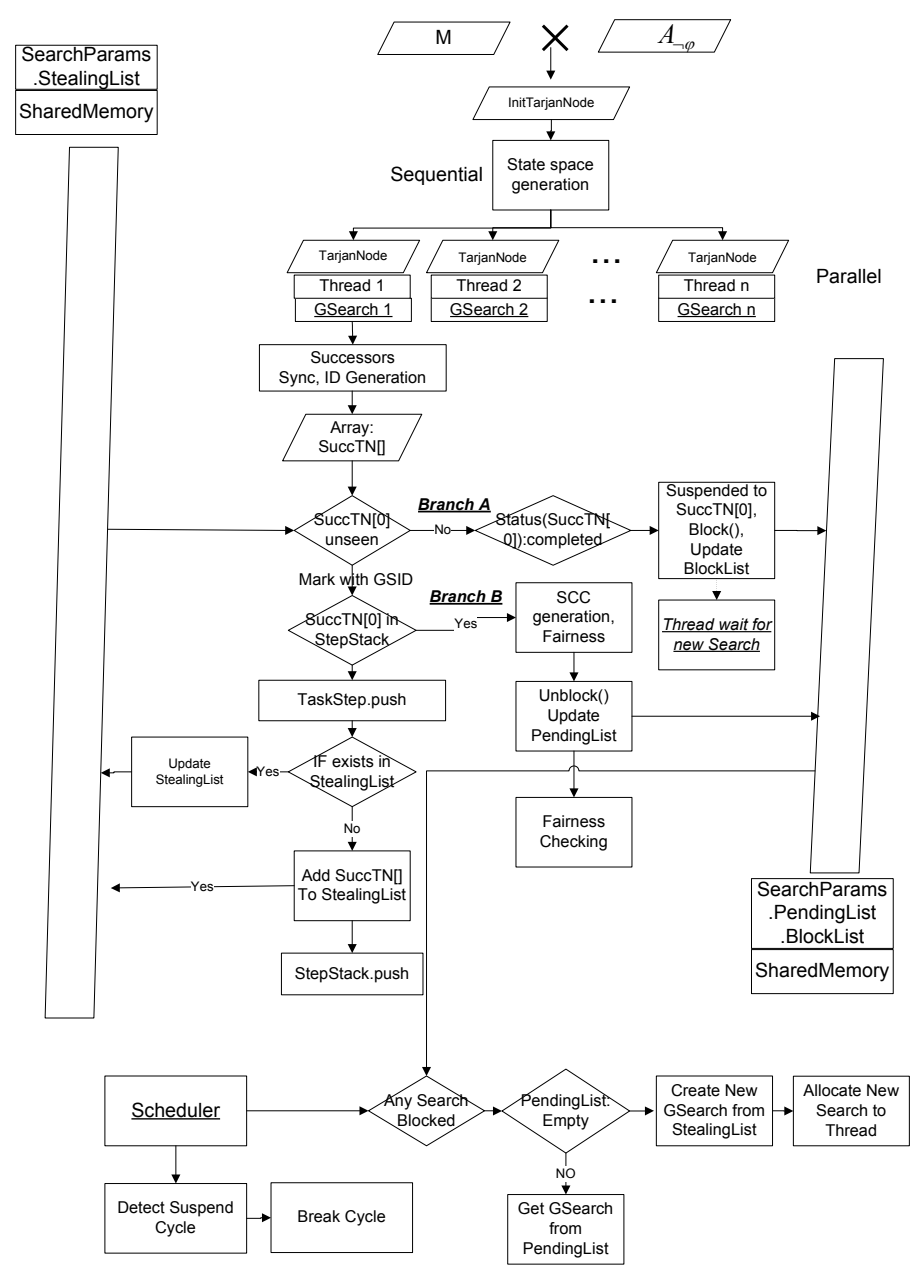

Figure 3. Overall Process

\section{A. Overview of our approach}

We introduce the overall execution process of our approach in Fig. 3. For readability, the figure shows only the main procedure of our approach. We present the complete algorithm later in this section.

First, we describe some essential concepts in our approach: (1) We define GSearch as our scheduling unit. Different than Search in Fig. 1, GSearch is a combination of state space generation and exploration. Each GSearch has a GSID to identify itself. Each GSearch has its own TaskStack and StepStack. A GSearch also needs to record the status of itself, e.g., whether the GSearch is suspended to any state. (2) A Thread is the physical execution unit. A thread can handle multiple GSearches based on scheduling. (3) We define the TarjanNode to indicate the state generated on the fly by GSearch, which is a meta-state. In TarjanNode, we define the SID and GSID. SID is the identification of TarjanNode in global state space, which is constructed by the string concatenation of $S_{1} S_{2} \ldots S_{n} S_{A}$. GSID represents the GSearch that explores this TarjanNode. We also define the status, suspendlist, which refer to the concurrent Tarjan's algorithm. enableEvt and ParticipatingProcesses are defined for the fairness checking. (4) We define SearchParams as the shared space among all GSearches. It consists of StealingList, PendingList, BlockList, SCCList, SuspendMap 
and the generated state space OGTrans. StealingList is to store all the generated TarjanNodes. It is a unique hash structure for duplicate elimination since TarjanNodes are generated concurrently in all GSearches. BlockList stores all GSearches that have been suspended. PendingList stores the resumed GSearch, which is waiting for Scheduler to allocate the free thread for it. SuspendMap stores the suspended relationship. e.g., GSearch ${ }_{1}$ is suspended to TarjanNode 2 . visited set is also contained in SearchParams, which indicates the states that have been expanded. (5) We integrate the Scheduler from Lowe. It takes the charge of creating new GSearch and assigning it to a free thread. A thread is regarded as free if the GSearch it handles is suspended to any state. If there is no GSearch in PendingList, new GSearches are created by assigning new initial states. The GSID is maintained by Scheduler.

Within the multi-core environment, we start multiple GSearches for each thread at the beginning. Thus, we can see in Fig. 3, the startup sequential state space generation process is to generate an initial set of states for each GSearch. This is a difference from the concurrent Tarjan's algorithm with known state space. Fig. 3 shows the execution process of one GSearch. With initial TarjanNode, GSearch initiates the preorder for it and generates the successors by the parallel composition. The synchronization operation is needed if synchronized events exist. The generated successors should be transferred to the array of TarjanNode SuccTN[]. We initilize the SID and other variants in TarjanNode. SuccTN $[0]^{1}$ is the next TarjanNode to be expanded based on the rule of DFS. Thus, GSearch ${ }_{1}$ should judge whether SuccTN[0] has been seen in other GSearches by accessing the StealingList.If not, SuccTN[0] is marked with GSearch ${ }_{1}$ 's GSID. If SuccTN[0] is not in StepStack, it is pushed to the TaskStack. Only SuccTN[O] belongs to GSearch . $_{\text {. }}$ Other TarjanNodes in SuccTN[] should be directly transferred to StealingList. Duplicate elimination is handled with the unique SID. It should be noted that when a GSearch writes its GSID to SuccTN[0], it should also update the StealingList if SuccTN[0] exists in StealingList without the mark of any GSID. Then the initial TarjanNode should also be pushed to StepStack.

Branch $A$ in Figure 3 presents that if SSearch $_{1}$ detects that SuccTN[0] is marked with other GSearch's GSID, it checks the status of SuccTN[0]. If SuccTN[0] is not completed, GSearch is suspended to SuccTN[0]. It updates the SuspendMap and the BlockList, and detects if a blocking cycle exists. If this is the case, it blocks the cycle by transferring all related TarjanNode to another one GSearch (refer to Fig. 2 in Section II-C). GSearch $_{1}$ becomes blocked and waits to resume. When a blocked GSearch is resumed, it is transferred to PendingList. Scheduler works on two tasks: (1) When a GSearch is suspended and waiting for resuming, the corresponding thread becomes free. If the PendingList is empty, it creates a new GSearch for the free thread. 2) If the PendingList is not empty, it gets GSearch from PendingList and pushes it to the free thread.

Branch $B$ in Figure 3 is the common lowlink updating process and the condition that a cycle being detected. In our approach, the fairness checking starts concurrently with the generation of the complete SCC. After the generation of the

\footnotetext{
${ }^{1}$ here we give the example in the first iteration, hence we regard SuccTN[0] as unexpanded
}

complete SCC, the suspended relationship should be updated. All GSearches which are suspended to TarjanNode in the SCC are resumed and continue their exploration.

\section{B. Data distribution}

The data distribution in our approach for each search consists of two parts: startup distribution and runtime distribution.

Firstly, in the on-the-fly LTL model checking, the state space is not known in advance. It is generated during the product between $M$ and $\mathcal{B}_{\neg \varphi} . M$ is also the parallel composition of component LTSs. So at the beginning, we always just have one initial state. Based on Section II-C, in that algorithm, fixed number of threads are started for concurrent searches at the beginning, which start on different states to follow different traces to increase the possibility to find SCC. For our approach, we should also start multiple GSearches for high efficiency. The difference is that for on-the-fly generated state space, we first start the state space generation for several iterations to generate partial state space. Then we base on the number of cores in the working machine to start the corresponding number of threads and GSearches. So at the beginning, the parallelism can be fully utilized.

Secondly, during the concurrent search process in our approach, the data distribution is based on the state space generation. When GSearch is started, each GSearch marks its startup state with its GSID. Then each GSearch stores all generated successor states in its Stepstack and DFSstack. It should be noticed as the state space generation is a concurrent process in many threads, it is impossible to config the global ID to identify each successor state as [11] does. In our approach, we mention in Section II-A that each state is indeed composed of many states from component LTSs and the automaton, so we can use a string to identify the generated states, which is the montage of each states' ID in the original component LTS or the Büchi Automaton. As GSearch works on a DFS process, each GSearch only marks the front state in the DFSstack with its GSID, then all successors are copied to the StealingList and the outgoing relations are copied back to the table Outgoing Trans. The key point is that before marking any successor state, the GSearch should visit the StealingList to check if this successor state is visited or marked with other GSID, and then decide if the search should continue, backtrack or suspend.

\section{Concurrent State Space Generation}

In our approach, the state space is generated concurrently by all active GSearches. Each GSsearch handles the successor generation independently. During the successor generation, event synchronization operations are involved in both the parallel interleaving of $M_{i}$ and the composition between $M$ and $\mathcal{B}_{\neg \varphi}$. We allocate a private array for each GSearch to temporarily store the generated successors. Event synchronization also works on this array. When we convert each successor state $\left(s_{i}, b_{i}\right)$ to TarjanNode, we need to explore all $s_{i}$ to fill up the information for fairness checking. These are all independently handled by each GSearch. The key point is, the state space generation in the concurrent environment refers to a lot of operations on shared space. e.g., the SearchParam. Thus, it is important to handle the concurrent access to prevent any data inconsistency. 
In order to ensure the consistency of shared data, lock is used throughout the program. In general, each shared variable should have a separate lock to ensure that at one time, only one GSearch can access it. SearchParams, as we have mentioned in Section III-A, is the structure to store shared variables among GSearches. In order to ensure synchronization ${ }^{2}$, most collection variables in SearchParams are created as the concurrent collection data type, which is provided by C\#.NET. For other shared variables, the SearchParams has static locks to ensure their synchronization. In order to ensure synchronization and correctness of the program, we present some details of locks below, which indicate how errors occur without these locks.

- TarjanNode.visitLock: A TarjanNode is a shared variable. During the exploration of the state space, each GSearch checks the TarjanNode via the following steps: (a) Checks the status. (b) Checks whether it is the first unexpanded successor in GSearch. If yes, the GSearch visits the node. These steps are supposed to be atomic. Otherwise, two GSearches may take the same TarjanNode and cause errors, as shown in Fig. 4. Two GSearches are handled by two threads. The first GSearch is taking the TarjanNode and hasn't finished, while the other GSearch is also checking the ownership of the node. Two GSearches take the same node and none of them being suspended. Thus, visitedlock is necessary to lock these three steps.

- Suspendmap.Lockitself: The updating and accessing of the SuspendedMap should be synchronized, otherwise blocking cycle may be missed. For example, in Fig. 5, one GSearch is checking whether blocking cycle existed or not. When it gets the conclusion that no cycle exists, and it has not added new suspension into the map, other GSearch starts to check the SuspendedMap to get the path. Both of the GSearches think that there is no blocking cycle and add the suspension into the map. At this condition, blocking cycle occurs but no one handles it. Then deadlock occurs.

- TarjanNode.block() \& TarjanNode.unblock(): Two or more GSearches may become blocked on the same TarjanNode concurrently. Moreover, the TarjanNode may be detected in an SCC at the same time. Shown in Fig. 6, when the first GSearch is updating the status of the TarjanNode but not yet finished, the second GSearch is checking TarjanNode's GSID. The second GSearch thinks that the TarjanNode is incomplete and starts to block itself on this node. However, the first GSearch sets the node as complete and unblocks all blocked searches on this node. In this situation, the status of this node has already been completed and cannot go through the unblocking process again. The first GSearch cannot get a chance to be active again.

\section{On-the-fly Parallel Fairness Verification}

We introduce the definition of fairness assumption and our major efforts on on-the-fly parallel fairness checking in previous sections. Our fairness checking is based on the exploration of SCCs. When the concurrent LTL model checking algorithm detects the existence of an SCC, it needs to generate

\footnotetext{
${ }^{2}$ Different from event synchronization, this represents the synchronization of data.
}

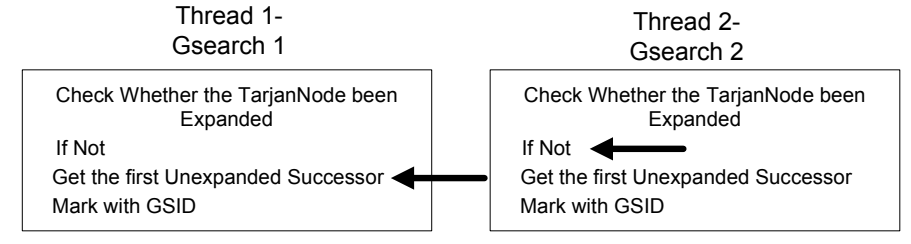

Figure 4. TarjanNode Synchronization

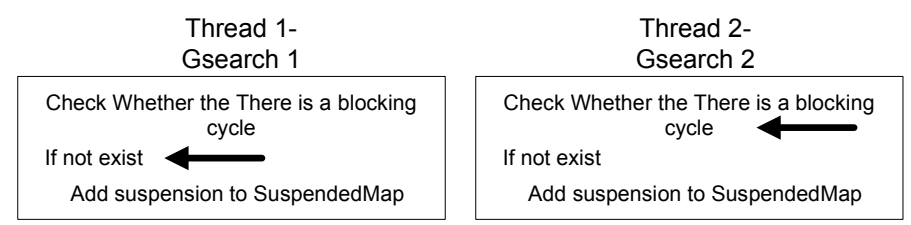

Figure 5. SuspendedMap Synchronization

the complete SCC. Shown in Fig 7, our key idea is to overlap part of the fairness checking process with the SCC generation process and do on-the-fly parallel checking so as to increase the performance. For instance, we overlap the generation of the sets enabledEvt, enabledPro, engagedEvt and engagedPro which base on the complete exploration of the SCC. Then we do parallel exploration to deal with these sets to generate the verification result. If any thread finds the condition that makes the SCC fair/unfair, the checking process terminates. In specific, Given an SCC $S c c$, we present the detail design for $E S F / P S F$ as a sample. We also specify the $S G F$.

Sequential Algorithm: The algorithm explores every component $s_{i}$ in $S c c$. For each state, the algorithm gets its engaged event list and add to the engagedEvt/Pro $(S c c)$. Then it explores each component again to find which component has any event $\alpha \in$ enabledEvt/Pro $\left(s_{i}\right)$ but $\notin$ engagedEvt/Pro $(S c c)$. These states are regarded as bad states. Then the algorithm removes these bad states from $S c c$ to get $S c c^{\prime}$, and calls the modified Tarjan's algorithm again to check whether there is an SCC in the $S c c^{\prime}$. If so, $S c c$ is fair. Or it is not fair.

Parallel On-the-fly Approach: engagedEvt/Pro(Scc) are generated during the generation of $S c c$. Bad states are generated and removed from the SCC concurrently with the lock. Each thread takes charge of several components of $S c c$. The new SCC is used to call the modified Tarjan's Model Checking algorithm. The modified Tarjan based model checking algorithm is not the concurrent version mentioned in Section II-B because the overhead generated by concurrency may be more expensive than the advantages provided as the size of a single SCC may be limited.

For SGF, the sequential algorithm explores every component $s_{i}$ in $S c c$. When $s_{i}$ is being explored, it generates the enabledEvt $\left(s_{i}\right)$, in which it contains a set of event ID. Then the algorithm explores all successors of $s_{i}$. For successors that also $\in S c c$, in the corresponding events that leads to the transition to these successors, if the ID of any event eid $\in$ enabledEvt $\left(s_{i}\right), S c c$ is fair. For SGF, there is no need to generate a set that based on the complete exploration of $S c c$, so this algorithm doesn't contain the on-the-fly part. In the parallel algorithm, shared memory is used to store $S c c$, and a fixed number of threads start to check all components. Checked 


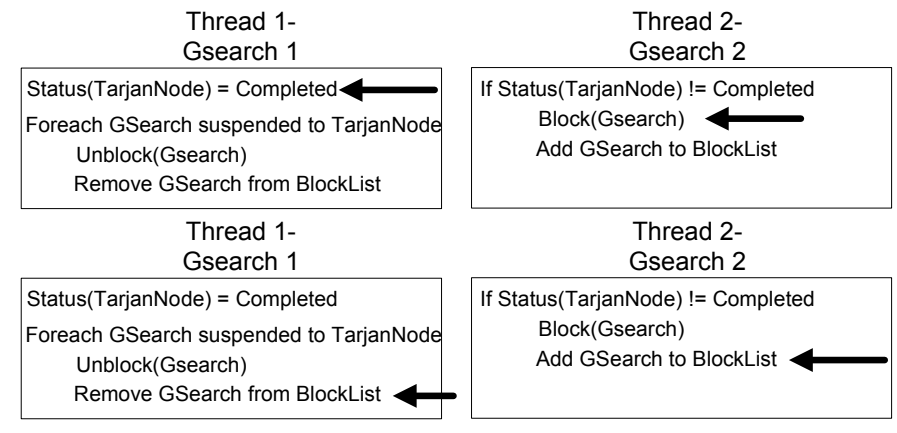

Figure 6. Block and Unblock GSearch Synchronization

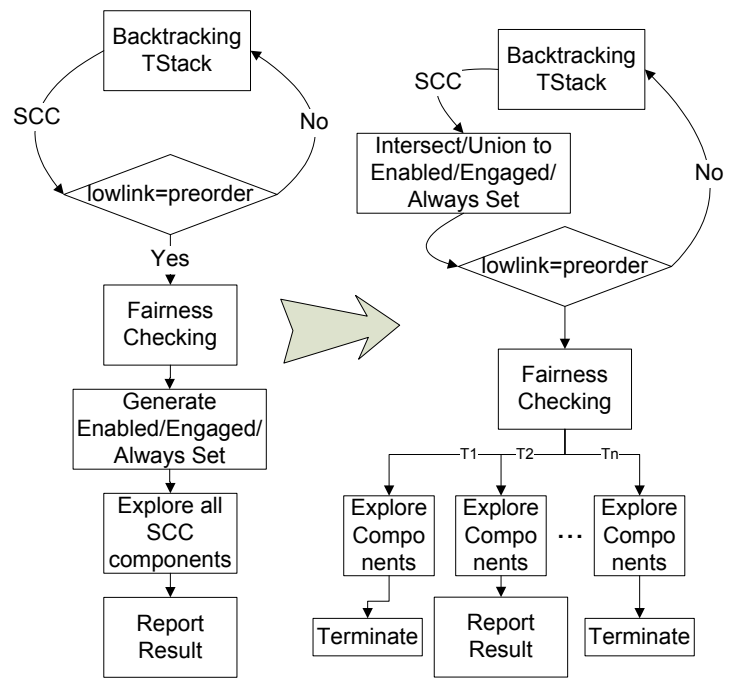

Figure 7. Parallel On-the-fly Fairness Checking

components are marked. If any thread finds the component that makes $S c c$ fair, it broadcasts to other threads and all thread terminate.

\section{E. Algorithm}

In this section, we present the algorithm of the concurrent on-the-fly SCC detection for LTL model checking with fairness assumption synthesizing our descriptions in the preceding sections. They are shown in Algorithm 2, Algorithm 3 and List. 1.

We describe the startup of our approach in Algorithm 2. SearchParams is defined in line 1. Line 2 is a Sequential state space generation processThis process generates some states which are stored in the StealingList. It should be guaranteed that no duplicates exists. Scheduler starts in Line 3 to create GSearch. The number of threads is same as the initial number of GSearches. All GSearches work concurrently.

We present the algorithm of GSearch in Algorithm 3 and List 1, which is the key algorithm of our approach. All operations are based on TarjanNode instead of the original state. In Algorithm 3, all GSearches access the shared memory to visit $M, \mathcal{A}_{\neg \varphi}$ and SearchParams. All key differences compared to sequential algorithm are shown with underline. Each GSearch has its own TaskStack and StepStack. In line 1, GSearch gets its initial state and converts it to a initial TarjanNode. The iteration for SCC detection starts in line 3. Line 4 is to set the
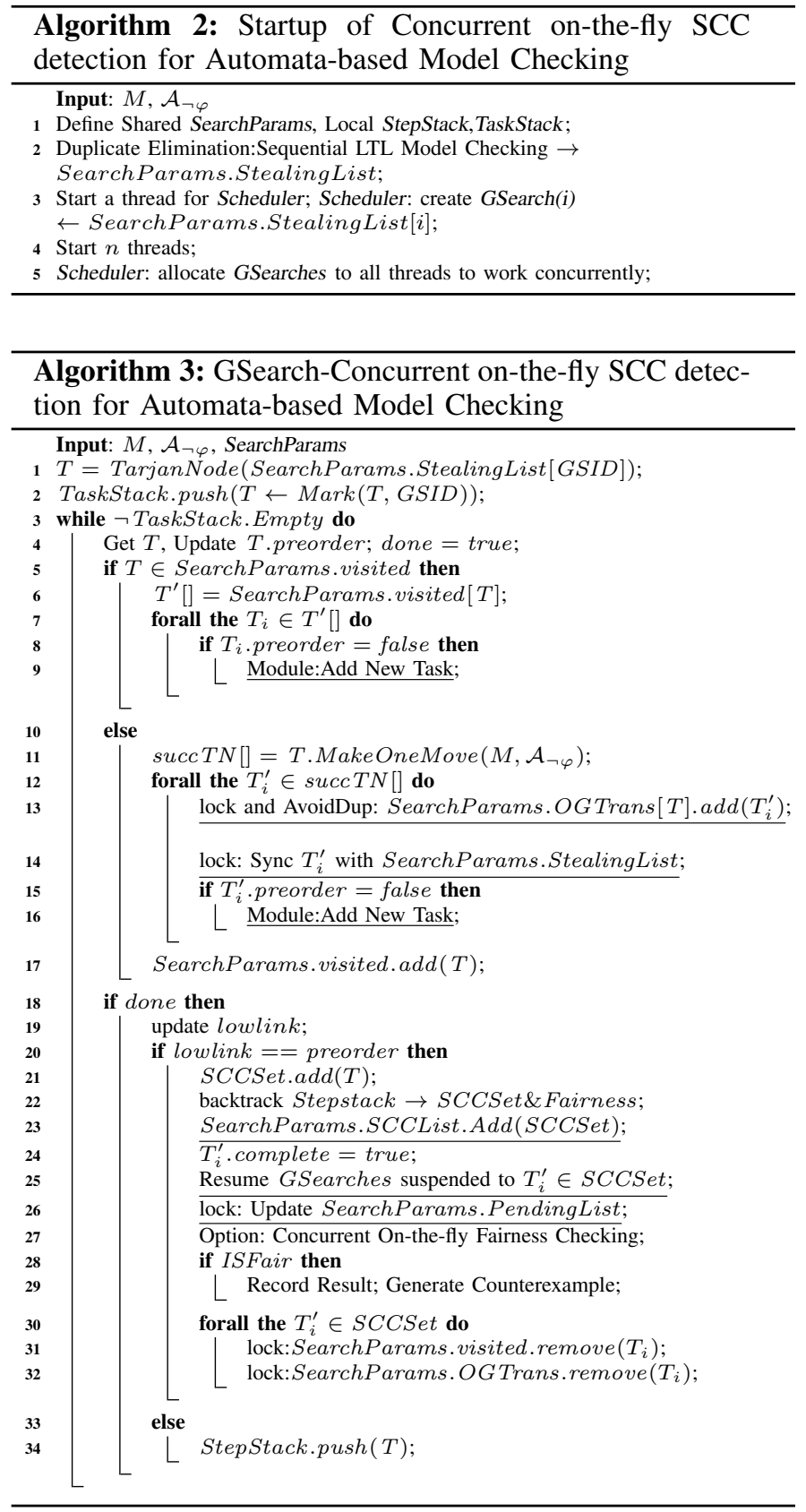

local preorder of TarjanNode in a certain GSearch. Line 5 and 10 lead to two conditions shown below:

(1) From line 5 to 9. If the TarjanNode $T$ is in SearchParams.visited, it has been expanded. Then in lines 6 to 9, GSearch gets and chooses the available successor to push into TaskStack, which is handled in the module Add New Task. We present that in List. 1. We judge whether the successor TarjanNode belongs to other GSearches in line 1. If not, we just mark the first available successor with GSID and push it into TaskStack in line 3 to 6 . If the successor already exists in SearchParams.StealingList but not been occupied by any GSearch, we should update its GSID. If the successor belongs to another GSearch and hasn't being completed, GSearch is suspended to this successor in lines 11 to 14 . It updates the SuspendedMap and detects whether this operation generates a 
blocking cycle. If so, it breaks this cycle, refers to Fig. 2 in Section II.

(2) From line 10 to 17. If the visiting TarjanNode $T$ is not expanded, GSearch generates successors based on $T$ by the interleaving between $M$ and $\mathcal{A}_{\neg \varphi}$ (line 11). It updates the OGTrans[T] without duplication (line 13). This operation should use lock to avoid conflicts. For each TarjanNode in SuccTN [], GSearch synchronizes the value of it with the data in StealingList (line 14). It checks whether the TarjanNode has been expanded before or been occupied by other GSearch. If it is a new TarjanNode without being marked with any GSID, the module Add New Task works as mentioned. Line 17 marks $T$ as expanded. During the process from line 5 to 17 , if all successors of $T$ have been expanded, it comes to line 19. It is to update the lowlink of $T$ and is same to the sequential algorithm.

GSearch generates an SCC in lines 20 to 26. The status of all TarjanNodes in SCC is marked as completed. All GSearches that being suspended to these TarjanNodes are resumed to be ready for scheduling. If fairness checking is required, the concurrent on-the-fly fairness checking works in line 27. It should be noted that in line 22, the fairness checking has started and overlaps with the generation of SCC. This is part of our concurrent on-the-fly fairness checking, which can improve the performance of fairness checking. If the detected SCC satisfies the fairness assumption, on-the-fly LTL model checking enters the counterexample generation process. Line 31 and 32 work as we always need to generate all SCCs. In line 34 , if the SCC is not detected, GSearch pushes $T$ to the local StepStack.

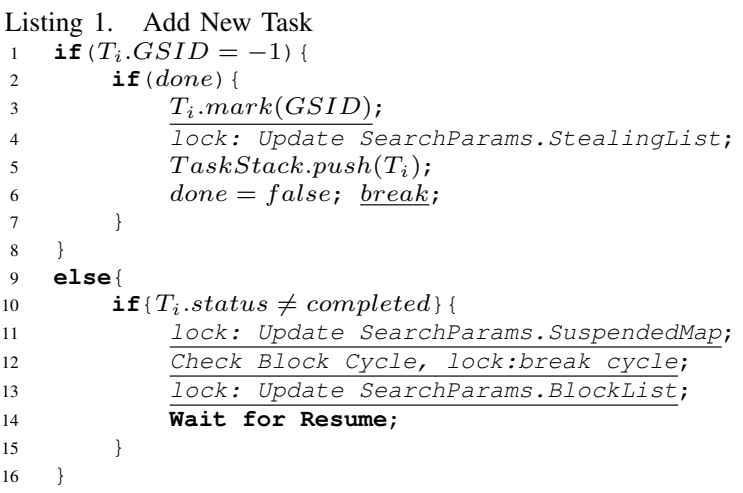

\section{F. Complexity}

In order to compare with sequential Tarjan's algorithm and Lowe's concurrent version with unrooted mode, We discuss the complexity of concurrent on-the-fly SCC detection without the complexity of state space generation. Given a transition system with $N$ nodes and $E$ edges, the sequential Tarjan's algorithm shows the complexity of $O(N+E)$ and Lowe shows the complexity of concurrent Tarjan's algorithm is $O\left(N^{2}+E\right)$. Our concurrent on-the-fly SCC detection has extra node transfer for the construction of global outgoing transition relationships and the StealingList mentioned before. The complexity of these part is $O(N)$ since we use lock and hash table to avoid duplicates. Thus, the complexity of our concurrent on-the-fly SCC detection without the complexity of state space generation is also $O\left(N^{2}+E\right)$.
Table I. Evaluation of LTL Model Checking (TIME IN SEC)

\begin{tabular}{|c|c|c|c|c|c|c|c|}
\hline Model & Proc & $|S|$ & $|T|$ & $|S C C s|$ & PAT-O & PAT-C & SP \\
\hline MsS & 1000 & $9 * 10^{3}$ & $1.8 * 10^{4}$ & 0 & 222.6 & 207.3 & 1.1 \\
\hline MsS & 1200 & $2.6 * 10^{4}$ & $5.3 * 10^{4}$ & 0 & 611.9 & 523.4 & 1.2 \\
\hline MsS & 1500 & $3.3 * 10^{4}$ & $6.7 * 10^{4}$ & 0 & 1104 & 626 & 1.76 \\
\hline CC & 4 & $1.5 * 10^{4}$ & $7 * 10^{4}$ & $5.2 * 10^{3}$ & 1.05 & 1.57 & 0.67 \\
\hline CC & 5 & $5 * 10^{5}$ & $3.3 * 10^{6}$ & $1.6 * 10^{5}$ & 49.7 & 31.9 & 1.6 \\
\hline ABP & 100 & $2.1 * 10^{5}$ & $7 * 10^{5}$ & 1 & 10.8 & 23.2 & 0.5 \\
\hline ABP & 200 & $8 * 10^{5}$ & $2.7 * 10^{6}$ & 1 & 53 & 92 & 0.58 \\
\hline ABP & 300 & $1.8 * 10^{6}$ & $6 * 10^{6}$ & 1 & 150.2 & 247 & 0.61 \\
\hline Lift.1 & 3,2 & $3.2 * 10^{5}$ & $7.2 * 10^{5}$ & 1349 & 39.6 & 24.7 & 1.6 \\
\hline Lift.2 & 3,2 & $3.8 * 10^{5}$ & $8.7 * 10^{5}$ & 1569 & 45.8 & 33.4 & 1.4 \\
\hline Lift.3 & 3,2 & $4.6 * 10^{5}$ & $1.5 * 10^{6}$ & 983 & 55.6 & 32.6 & 1.7 \\
\hline
\end{tabular}

\section{IMPLEMENTATION AND EVALUATION}

We implement our approach using $C \#$ in Process Analysis Toolkit (PAT) [17]. We call it PAT-C. We evaluate the performance of $P A T-C$ by comparing it to the original PAT with sequential on-the-fly LTL model checking and fairness checking, which we call $P A T-O$ and $P A T-O F$. We conduct our experiments on a laptop with Intel(R) Xeon(R) CPU E5-1650, 3.2GHZ, 12 cores, 16GB RAM.

In our experiments, $|S|,|T|$ and $|S C C s|$ separately represent the number of states, the number of transitions and the number of SCCs. $L S C C$ and $S S C C$ separately represent the size of the largest SCC and smallest SCC. SP means the speedup. For the testing models, MsS represents the Miners Scheduler. CC represents Consensus with Crashes. DP represents Dining Philosophers. ABP represents Alternation Bit Protocol. KvR.1/2 represents K-valued Register.12. MLS.12 represents Multiple Lift Sysmtem 12. TBM represents DBM Testing.

\section{A. Performance Evaluation on SCC Detection for Model Checking}

We conduct the performance evaluation on multiple LTS models on a range of state space. We initialize 12 GSearches. We start 12 threads in parallel to handle these GSearches concurrently since our machine owns 12 cores. It should be noted that in order to completely evaluate the performance of our approach, in our experiments we find all SCCs instead of just one. We involve four models in this part: $M s S, C C$, $A B P$ and Lift. Our experimental results are shown in Table I: (1) The product between $M s S$ model and the Büchi Automaton generates no SCCs. Hence, our approach works as a concurrent complete state space generation process. We use 1000, 1200 and 1500 processes for our experiments. From Table I, we can observe: (1) Our approach gains up to $2 \mathrm{X}$ speedup compared to the sequential LTL model checking, which is significant since the original execution cost is fairly large. 2) The product between CC\&Lift.1/2/3 model and the Büchi Automaton can generate a large number of small SCCs. Results in Table I show that with a large number of small SCCs, our approach can also gain around $2 \mathrm{X}$ speedup compared to the sequential LTL model checking in PAT. 3) The product between ABP model and the Büchi Automaton generates just one large size SCC. Results in Table I show that the performance with these kind of models is expected to be even slower than the sequential LTL model checking in PAT.

In conclusion, our approach can generally improve the performance of automata-based LTL model checking. The performance is better for larger state spaces. The performance 
Table II. Testing Model for FAIRnEss CHECKING

\begin{tabular}{|c|c|c|c|c|c|c|}
\hline Model & Proc & $|S|$ & $|T|$ & $|S C C s|$ & LSCC & SSCC \\
\hline DP & 8 & $1.4 * 10^{5}$ & $1.08 * 10^{6}$ & 51 & $3 * 10^{4}$ & 1 \\
\hline DP & 9 & $5.3 * 10^{5}$ & $4.5 * 10^{6}$ & 66 & $1.1 * 10^{5}$ & 1 \\
\hline DP & 10 & $1.9 * 10^{6}$ & $1.8 * 10^{7}$ & 83 & $4.2 * 10^{5}$ & 1 \\
\hline Peterson & 4 & $5.1 * 10^{4}$ & $2.1 * 10^{5}$ & 5 & $4.5 * 10^{4}$ & 1 \\
\hline Peterson & 5 & $1.4 * 10^{6}$ & $7.2 * 10^{6}$ & 17 & $1.3 * 10^{6}$ & 1 \\
\hline CC & 4 & $1.2 * 10^{4}$ & $5.4 * 10^{4}$ & $3.8 * 10^{3}$ & 2 & 1 \\
\hline CC & 5 & $3.8 * 10^{5}$ & $2.5 * 10^{6}$ & $1.2 * 10^{5}$ & 2 & 1 \\
\hline KvR.2 & 4,3 & $6.2 * 10^{5}$ & $2.5 * 10^{6}$ & 1042 & $4.1 * 10^{5}$ & 104 \\
\hline KvR.2 & 5,3 & $3.7 * 10^{6}$ & $1.5 * 10^{7}$ & 2748 & $2.3 * 10^{6}$ & 256 \\
\hline KvR.2 & 4,4 & $3.8 * 10^{6}$ & $1.8 * 10^{7}$ & 6342 & $2.4 * 10^{6}$ & 104 \\
\hline MLS.1 & $2,2,3$ & $3.8 * 10^{5}$ & $1.9 * 10^{6}$ & 940 & $2.7 * 10^{5}$ & 4 \\
\hline MLS.1 & $3,2,2$ & $1.2 * 10^{6}$ & $5.1 * 10^{6}$ & 1416 & $9.8 * 10^{5}$ & 4 \\
\hline MLS.1 & $2,2,4$ & $1.5 * 10^{6}$ & $9.3 * 10^{6}$ & 3243 & $1.1 * 10^{6}$ & 4 \\
\hline MLS.2 & $2,2,3$ & $6.5 * 10^{5}$ & $3.5 * 10^{6}$ & 3029 & $3.5 * 10^{5}$ & 1 \\
\hline MLS.2 & $3,2,2$ & $2.2 * 10^{6}$ & $9.5 * 10^{6}$ & 5457 & $1.5 * 10^{6}$ & 1 \\
\hline MLS.2 & $2,2,4$ & $2.7 * 10^{6}$ & $1.6 * 10^{7}$ & 8345 & $1.4 * 10^{6}$ & 1 \\
\hline DBM & 2,4 & $4.2 * 10^{4}$ & $1.7 * 10^{6}$ & 1 & $1.4 * 10^{4}$ & - \\
\hline DBM & 2,5 & $9.5 * 10^{4}$ & $4.5 * 10^{6}$ & 1 & $3.2 * 10^{4}$ & - \\
\hline DBM & 2,6 & $1.9 * 10^{5}$ & $1.1 * 10^{7}$ & 1 & $6.3 * 10^{4}$ & - \\
\hline PMC & 50,1000 & $1 * 10^{6}$ & $2 * 10^{6}$ & 51 & $2 * 10^{4}$ & $2 * 10^{4}$ \\
\hline PMC & 60,1000 & $1.2 * 10^{6}$ & $2.4 * 10^{6}$ & 61 & $2 * 10^{4}$ & $2 * 10^{4}$ \\
\hline PMC & 80,1000 & $1.6 * 10^{6}$ & $3.2 * 10^{6}$ & 81 & $2 * 10^{4}$ & $2 * 10^{4}$ \\
\hline
\end{tabular}

decreases at $A B P$ model since it has only one large SCC in the state space. Thus, based on our approach, all GSearches easily encounter TarjanNode in other GSearches. According to the cycle breaking rules, our approach works like a sequential algorithm as all data is transferred to one GSearch. The parallelism cannot be well utilized and a lot of time is consumed at transferring nodes. Finally the concurrent on-the-fly model checking is more suitable for the models with a large amount SCCs and trivial average SCC size so as to reduce the cost of suspending, which is also indicated in [11].

\section{B. Performance of On-the-fly Parallel Fairness Verification}

We conduct the experiments on fairness checking using different models with different SCC numbers and SCC sizes. For each type of fairness, we choose three models and we perform the experiments independently. The features of our test models are shown in Table II. These models differ in the number and size of SCCs, which can help reflect the features of our approach. In this part, the parallelism started is less then 11 threads, which is adjusted dynamically based on the scheduling of the Microsoft .Net platform.

In our experiments, we measure the time cost in milliseconds. Firstly, we compare our approach to the sequential fairness checking in PAT. The results of ESF, EWF, PWF and $P S F$ checking is shown in Table III. We can see that our approach for fairness checking can gain significant speedup for most models ${ }^{3}$. The performance improvement is more visible when the state space consists of a lot of SCCs and the average SCC size is large. A performance decrease is observed at the $C C$ model because all SCCs in the state space are very small, with the size of 1 or 2. For Peterson, where the state space consists of just $5 \mathrm{SCCs}$, the performance is also decreased. Under this condition, the overhead of parallelism is more dominant than the advantage of parallelism. Secondly, as we mentioned in Section III-E, our approach generates the sets for fairness checking together with the SCC generation, so it introduces costs to the SCC generation process. We count the cost of the entire SCC generation and Fairness checking for some models, which is shown in the right side of " / in

\footnotetext{
${ }^{3}$ Here all models represent the state space generated from the product between the model and the Büchi Automaton.
}

Table III. Evaluation of ESF\&EWF CHECKING (TIME IN MS)

\begin{tabular}{|c|c|c|c|c|c|c|c|}
\hline \multirow{2}{*}{ Model } & \multirow{2}{*}{ Proc } & \multicolumn{3}{|c|}{ ESF Checking } & \multicolumn{3}{c|}{ EWF Checking } \\
\cline { 3 - 8 } & & PAT-OF & PAF-C & SP & PAT-OF & PAF-C & SP \\
\hline DP & 8 & $1.1 / 1.5 * 10^{3}$ & $48 / 304$ & $25 / 5$ & - & - & - \\
\hline DP & 9 & $1.5 / 1.6 * 10^{4}$ & $81 / 1218$ & $188 / 13$ & - & - & - \\
\hline Peterson & 4 & 29 & 30 & 0.97 & - & - & - \\
\hline Peterson & 5 & $1.1 * 10^{3}$ & 267 & 4.3 & - & - & - \\
\hline CC & 4 & 3 & 3 & 1 & 3 & 95 & - \\
\hline CC & 5 & 137 & 157 & 0.87 & 157 & 3415 & - \\
\hline KvR.2 & 4,3 & - & - & - & 119 & 4 & 29.7 \\
\hline KvR.2 & 5,3 & - & - & - & 716 & 16 & 44.8 \\
\hline KvR.2 & 4,4 & - & - & - & 750 & 38 & 19.7 \\
\hline
\end{tabular}

Table IV. EVAluation of PSF\&PWF ChECKING (TIME IN MS)

\begin{tabular}{|c|c|c|c|c|c|c|c|}
\hline \multirow{2}{*}{ Model } & \multirow{2}{*}{ Proc } & \multicolumn{3}{|c|}{ PWF Checking } & \multicolumn{3}{|c|}{ PSF Checking } \\
\hline & & PAT-OF & & $\mathrm{SP}$ & $\begin{array}{l}\text { PAT-OF } \\
\end{array}$ & PAF-C & $S P$ \\
\hline DP & 8 & 50 & 1 & 50 & $1327 / 1705$ & $14 / 364$ & $95 / 5$ \\
\hline DP & 9 & 224 & 1 & 224 & $1.7 / 1.8 * 10^{4}$ & $48 / 1497$ & $364 / 11$ \\
\hline MLS.1 & $2,2,3$ & $153 / 1728$ & $4 / 1270$ & $38 / 1.4$ & 187 & 71 & 2.6 \\
\hline MLS.1 & $3,2,2$ & $892 / 6100$ & $3 / 5100$ & $298 / 1.2$ & 617 & 161 & 3.8 \\
\hline MLS.1 & $2,2,4$ & $733 / 7256$ & $9 / 5453$ & $81 / 1.35$ & 819 & 288 & 2.9 \\
\hline MLS. 2 & $2,2,3$ & $257 / 2797$ & $4 / 2019$ & $64 / 1.3$ & 340 & 219 & 1.6 \\
\hline MLS.2 & $3,2,2$ & $1432 / 12601$ & 19/10613 & $77 / 1.2$ & $1.1 * 10^{3}$ & 475 & 2.4 \\
\hline MLS.2 & $2,2,4$ & $1290 / 13382$ & $16 / 11800$ & $80 / 1.2$ & $1.4 * 10^{3}$ & 833 & 1.7 \\
\hline
\end{tabular}

Table III. We can see that our approach can still gain significant speedup. The reason why the speedup is not as high as the results in the left side of " $"$ is that the SCC generation cost a lot compared with the fairness checking. It can also be concluded that the parallelization of fairness checking can bring more performance improvements for weak fairness type than strong fairness type. The reason is that the weak fairness checking does not have to update any shared variables(bad states), therefore lock operation is not necessary. In contrast, in strong fairness checking, a lock exists. During the execution, some threads need to wait for other threads for the right to access.

The results of SGF checking is shown in Table V. For SGF checking, as we mentioned in Section III-E, the complete checking process works in parallel after the generation of SCC. Hence, we just record the fairness checking time and compare it with the sequential version. We can see besides the $C C$ model with very small SCC, our approach gains around $5 \mathrm{X}$ speedup for the $D B M$ and around $4 \mathrm{X}$ speedup for the $P M C$.

Finally, we compare our experimental results with the previous results of Liu, Sun and Dong [10], which also works on the parallel on-the-fly LTL model checking in PAT. In [10], the major Tarjan process is taken in one thread and SCC generation is taken in parallel by forking new threads to handle it. The performance of both the approach in [10] and this paper depends on the ratio of SCCs and the average SCC size. The differences are that the on-the-fly LTL model checking in [10] works better than sequential algorithm only with large number of large size SCCs. Our approach can also gain performance improvement without any SCC, which is not possible for the approach in [10]. The large number of small size SCCs may influence the fairness checking process in both of these approaches. But for large sized SCCs, the approach shown in this paper may have better performance improvement than PAT as we start the exploration from different directions and the process is on-the-fly. In conclusion, the approach in this paper can be suitable for much more types of models and gain more performance improvement than the approach in [10].

\section{CONClusion ANd Future Work}

In this paper, we expanded the concurrent Tarjan's algorithm and developed a concurrent on-the-fly SCC detection for 
Table V. SGF CHECKING (TIME IN MS)

\begin{tabular}{|c|c|c|c|c|}
\hline Model & Proc & PAT-OF & PAT-C & SP \\
\hline DBM & 2,4 & $1.7 * 10^{3}$ & 353 & 4.8 \\
\hline DBM & 2,5 & $5.4 * 10^{3}$ & $1.1 * 10^{3}$ & 4.95 \\
\hline DBM & 2,6 & $1.5 * 10^{4}$ & $2.9 * 10^{3}$ & 5.1 \\
\hline PMC & 50,1000 & $1.1 * 10^{3}$ & 370 & 3.2 \\
\hline PMC & 60,1000 & $1.7 * 10^{3}$ & 453 & 3.7 \\
\hline PMC & 80,1000 & $1.9 * 10^{3}$ & 605 & 3.1 \\
\hline CC & 4 & 1 & 83 & - \\
\hline CC & 5 & 77 & $2.7 * 10^{3}$ & - \\
\hline
\end{tabular}

automata-based (LTL) model checking with fairness checking. To this end, we built a novel abstract data structure for concurrent LTL model checking taking data consistency into account. In addition, we developed a parallel on-the-fly fairness checking approach for different types of fairness assumptions. We also implemented our proposed approach in the practical model checker PAT. Our experiments show that our approach achieves significant speedup comparing to the sequential version of PAT. In the future, we plan to transfer our approach work to many-core (GPU) platform, which support massive parallelism and promises to accelerate the performance of model checking.

\section{REFERENCES}

[1] W. Anton and B. Dragan. GPUexplore: Many-Core On-the-Fly State Space Exploration Using GPUs. In TACAS, pages 233-247. 2014.

[2] C. Baier, J.-P. Katoen, et al. Principles of model checking, volume 26202649. MIT press Cambridge, 2008.

[3] V. Bloemen, A. Laarman, and J. van de Pol. Multi-core on-the-fly SCC decomposition. In PPoPP, page 8. ACM, 2016.

[4] E. M. Clarke, W. Klieber, M. Nováček, and P. Zuliani. Model checking and the state explosion problem. In Tools for Practical Software Verification, pages 1-30. 2012.

[5] J.-M. Couvreur. On-the-fly verification of linear temporal logic. In FM99tFormal Methods, pages 253-271. Springer, 1999.

[6] S. Evangelista, L. M. Kristensen, and L. Petrucci. Multi-threaded explicit state space exploration with state reconstruction. In ATVA, pages 208-223. Springer, 2013.

[7] S. Evangelista, A. Laarman, L. Petrucci, and J. Van De Pol. Improved multi-core nested depth-first search. In ATVA, pages 269-283. Springer, 2012.

[8] S. Evangelista, L. Petrucci, and S. Youcef. Parallel nested depth-first searches for LTL model checking. In ATVA, pages 381-396. Springer, 2011.

[9] L. Lamport. Proving the correctness of multiprocess programs. TSE, (2):125-143, 1977

[10] Y. Liu, J. Sun, and J. S. Dong. Scalable multi-core model checking fairness enhanced systems. In Formal Methods and Software Engineering, pages 426-445. Springer, 2009.

[11] G. Lowe. Concurrent Depth-First Search Algorithms. In TACAS, pages 202-216. 2014.

[12] G. Lowe. Concurrent depth-first search algorithms based on Tarjans Algorithm. STTT, 18(2):129-147, 2016.

[13] J. Pang, Z. Luo, and Y. Deng. On automatic verification of selfstabilizing population protocols. Frontiers of Computer Science in China, 2(4):357-367, 2008.

[14] A. Puhakka and A. Valmari. Liveness and fairness in process-algebraic verification. In CONCUR, pages 202-217. Springer, 2001.

[15] E. Renault, A. Duret-Lutz, F. Kordon, and D. Poitrenaud. Parallel explicit model checking for generalized Büchi automata. In TACAS, pages 613-627. Springer, 2015.

[16] J. Sun, Y. Liu, J. S. Dong, and J. Pang. Towards a Toolkit for Flexible and Efficient Verification under Fairness. Technical report, Technical Report TRB2/09, National Univ. of Singapore, 2008.

[17] J. Sun, Y. Liu, J. S. Dong, and J. Pang. PAT: Towards Flexible Verification under Fairness. In $C A V$, pages 709-714, 2009.
[18] R. Tarjan. Depth-first search and linear graph algorithms. SIAM journal on computing, 1(2):146-160, 1972.

[19] Z. Wu, Y. Liu, Y. Liang, and J. Sun. GPU Accelerated Dynamic Counterexample Generation in LTL Model Checking. In ICFEM, pages 413-429, 2014.

[20] Z. Wu, Y. Liu, J. Sun, J. Shi, and S. Qin. Gpu accelerated on-the-fly reachability checking. In ICECCS, pages 100-109. IEEE, 2015.

[21] L. Yang. Model checking concurrent and real-time systems: the PAT approach. PhD thesis, 2009. 